\title{
Influence of survivin and Bcl-2 expression on the biological behavior of non-small cell lung cancer
}

\author{
QIN GAO $^{1 *}$, SHENG YANG $^{2^{*}}$ and MING-QIANG KANG ${ }^{1}$ \\ Departments of ${ }^{1}$ Thoracic Surgery, and ${ }^{2}$ Oncology, The Union Hospital of \\ Fujian Medical University, Fujian 350001, P.R. China
}

Received September 25, 2011; Accepted December 23, 2011

DOI: $10.3892 / \mathrm{mmr} .2012 .840$

\begin{abstract}
Survivin and Bcl-2 are generally considered to be inhibitors of apoptosis and are frequently overexpressed in several types of human cancers. However, their role in regulating the biological behavior of non-small cell lung carcinoma (NSCLC) remains controversial. We aimed to determine the expression of survivin and Bcl-2 and explore their correlation with clinicopathological features and prognosis. The expression of survivin and $\mathrm{Bcl}-2$ proteins in 62 specimens of NSCLC tissues and 30 specimens of tumor adjacent tissues was detected using immunohistochemistry. The correlation between protein expression and clinicopathological features and prognosis was analyzed. The percentage of survivinpositive samples obtained from NSCLC tissues was $58.06 \%$ (36/62), which was significantly higher compared to that in normal lung tissues $(10 \%, 3 / 30 ; \mathrm{P}<0.05)$. Similarly, the percentage of Bcl-2-positive samples obtained from NSCLC tissues was statistically higher compared to that from normal lung tissues $(51.61 \%, 32 / 62$ vs. $6.67 \%, 2 / 30$; $\mathrm{P}<0.05)$. Survivin expression was closely correlated with tumor differentiation, lymph node metastasis and TNM stage $(\mathrm{P}<0.05)$, while Bcl-2 expression was only associated with TNM stage $(\mathrm{P}<0.05)$. The expression of survivin was positively correlated with that of Bcl-2 $(\mathrm{P}<0.05)$. A five-year follow-up study revealed that the expression of survivin and Bcl-2 was negatively correlated with post-operative survival duration. Our findings suggest that survivin and Bcl-2 may act synergistically in the occurrence, development, invasion and metastasis of NSCLC, both of which are up-regulated in NSCLC tissues. The co-expression of survivin and $\mathrm{Bcl}-2$, which is closely related to malignancy, may serve as a biomarker for predicting prognosis.
\end{abstract}

Correspondence to: Professor Ming-Qiang Kang, Department of Thoracic Surgery, The Union Hospital of Fujian Medical University, Fuzhou, Fujian 350001, P.R. China

E-mail: mingqiangkang@126.com

${ }^{*}$ Contributed equally

Key words: non-small cell lung cancer, survivin, Bcl-2, immunohistochemistry, prognosis, biological behavior

\section{Introduction}

Lung cancer, with a high incidence and mortality rate, is regarded as a serious threat to public health. The prevalence of lung cancer in China is increasing, whereas its pathogenesis has yet to be elucidated, and effective measures of prevention are still lacking (1). Recent studies concerning the molecular biology of tumors have revealed that the tumorigenesis and development of lung cancer is a complex biological process, involving a variety of genes and factors. The activation of oncogenes, or the inactivation of tumor-suppressor genes, promotes the abnormal proliferation of tumor cells, reduces tumor cell apoptosis and is therefore considered a key event in tumorigenesis, development, invasion and metastasis of lung cancer (2). Accumulating evidence has indicated that survivin and Bcl-2, both of which are apoptosis-suppression proteins, are closely associated with the biological behavior of various malignant tumors, including breast cancer, hepatocellular carcinoma and cervical cancer (3-5). In the present study, the expression of survivin and Bcl-2 in non-small cell lung cancer (NSCLC) tissues was evaluated using immunohistochemistry. We also explored their correlation with clinicopathological features and prognosis. Our results promote the further understanding of NSCLC pathogenesis and provide a scientific and theoretical basis for gene-based prognosis evaluation and gene therapy.

\section{Materials and methods}

Clinical materials. A total of 62 archival paraffin-embedded tissue specimens, obtained from 62 NSCLC patients who underwent surgical excision at the Department of Thoracic Surgery at the Union Hospital Affiliated Fujian Medical University between January 2001 and June 2005, were randomly selected. Approval was provided by the ethics committee of Fujian Medical University [reference no. 2000(24)]. Written consent was obtained from each patient or their relative before operation. Clinical stage was determined according to the new UICC TNM classification published in 1997 (6), and histological typing was determined according to the World Health Organization (WHO) criteria (7). Among these 62 cases, 44 were male and 18 were female. The ratio of male to female was $2.4: 1$. The patient age ranged from 35 to 78 years (mean 57.8 ). Thirty-two cases were $<60$ years of age, while 30 cases were $\geq 60$ years. Histological typing revealed that 40 cases were diagnosed as 


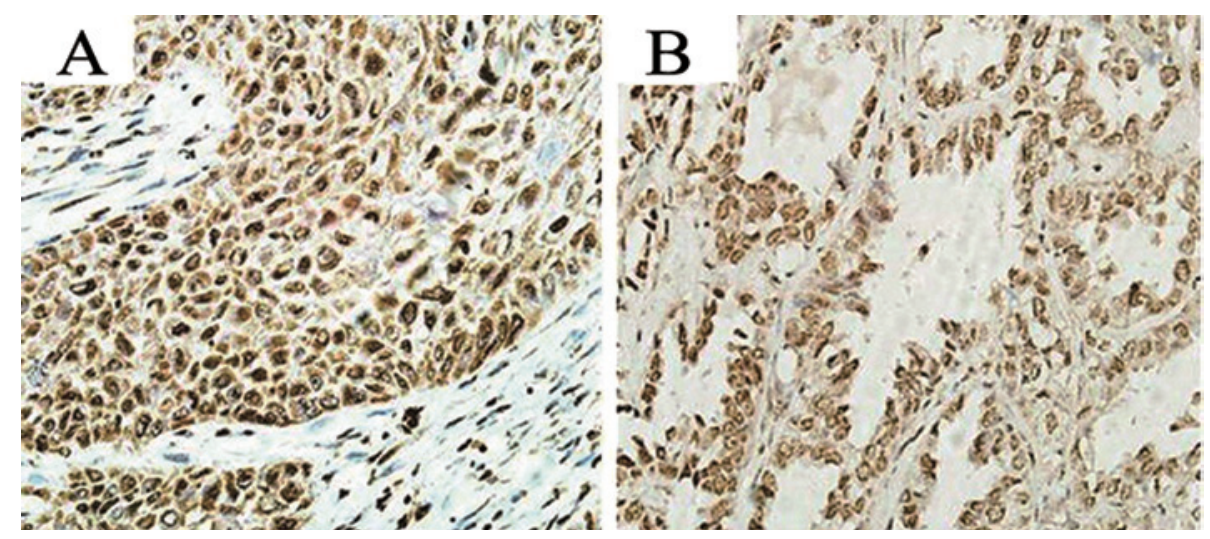

Figure 1. Representative immunohistochemistry for Survivin in non-small cell lung cancer (S-P; magnification, x400). (A) Squamous cell carcinoma; (B) adenocarcinoma.

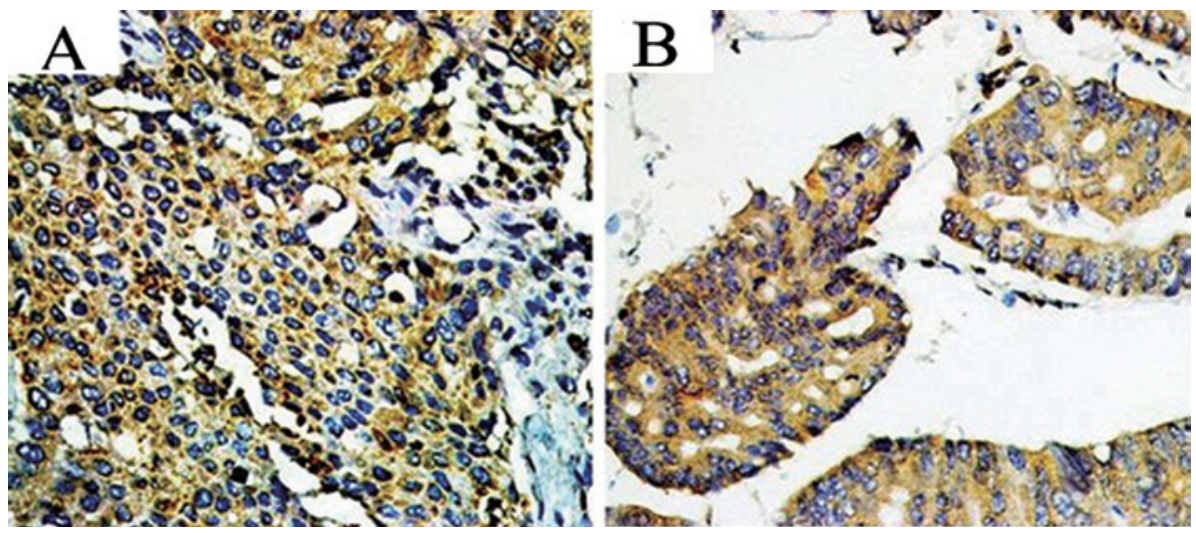

Figure 2. Representative immunohistochemistry for Bcl-2 in non-small cell lung cancer (S-P; magnification, x400). (A) Squamous cell carcinoma; (B) adenocarcinoma.

squamous cell carcinoma, 18 were adenocarcinoma and 4 cases were large-cell lung carcinoma. The tumor diameter was $<5 \mathrm{~cm}$ in 28 cases, while in 34 cases the tumor diameter was $\geq 5 \mathrm{~cm}$. Thirty-seven cases showed high or moderate tumor differentiation, while 25 cases showed poor differentiation. Lymph node metastasis was detected in 38 cases. According to TNM stage classification, 33 cases were stage I + II, and 29 were stage III + IV. None of the patients had received radiochemotherapy or chemotherapy prior to surgery. Meanwhile, 30 tissue specimens of tumor adjacent tissues, which were pathologically confirmed as $>5 \mathrm{~cm}$ away from the tumor tissues, were used as the control. The patients were followed up for 3-120 months. The survival duration of patients ranged from 3 to 98 months, and the median survival time was 36 months.

Reagents and methods. Formalin-fixed (10\%) samples were dehydrated and paraffin-embedded according to standard histological protocols. All the samples were sectioned into serial 4- $\mu \mathrm{m}$ slices. Streptavidin-peroxidase (S-P)-based immunohistochemistry was used to detect the protein expression of survivin and Bcl-2. Rabbit anti-human polyclonal anti-survivin antibody, mouse anti-human monoclonal anti-Bcl-2 antibody and the immunohistochemistry S-P kit were purchased from Fuzhou Maixin Biotechnology Development Co., Ltd. Staining was performed according to the manufacturer's instructions. For the negative control, phosphate-buffered saline (PBS) was applied instead of the primary antibody. Xylene, anhydrous ethanol and PBS were all of analytical grade.
Scoring methods. The immunostaining of survivin and Bcl-2 was scored as described by Sinicrope et al (8). Cells exhibiting yellow or brown-yellow vesicles in the cytoplasm or nuclear compartments were identified as immuno-positive. The weighted score for each tumor specimen was determined by the following formula: $\mathrm{H}=(\mathrm{N} x \mathrm{I})$. An $\mathrm{H}$ value of $>1$ was recognized as positive. Five fields were randomly selected under a microscope (magnification, x400) and the number of positive cells were counted. The percentage of immunopositive cells $(\mathrm{N})$ was assigned to one of five categories: $<5 \%$; $5-25 \% ; 25-50 \% ; 50-75 \%$; or $>75 \%$. The intensity of Bcl-2 and p53 immunostaining (I) was scored as: + , weak; ++ , moderate; or +++ , intense.

Statistical analysis. Data analyses were performed using SPSS 17.0. The difference between groups was evaluated by the Chi-square test for categorical data. Correlation was determined by Spearman analysis. Survival rate was calculated using the Kaplan-Meier method. The difference in survival rate was analyzed by the log-rank test. The level of significance was set at $\mathrm{P}<0.05$.

\section{Results}

Protein expression of survivin and Bcl-2 in NSCLC tissues and tumor adjacent tissues. As shown in Figs. 1 and 2, the immunostaining for survivin and Bcl-2 was detected in the cytoplasm or nucleus of tumor cells in the NSCLC tissues. The 
Table I. Expression of survivin and Bcl-2 in NSCLC and tumor adjacent tissues.

\begin{tabular}{lccccccc}
\hline & $\mathrm{n}$ & Survivin-positive $(\mathrm{n}, \%)$ & $\chi^{2}$ & P-value & Bcl-2-positive (n, \%) & $\chi^{2}$ & P-value \\
\hline NSCLC tissues & 62 & $36(58.06)$ & 19.125 & 0.00 & $32(51.61)$ & 17.530 & 0.00 \\
Tumor adjacent tissues & 30 & $3(10.00)$ & & & $2(6.67)$ & & \\
\hline
\end{tabular}

Table II. Relationship between survivin and Bcl-2 expression and clinicopathological features of NSCLC.

\begin{tabular}{|c|c|c|c|c|c|c|c|c|c|}
\hline \multirow{2}{*}{$\begin{array}{l}\text { Clinicopathological } \\
\text { features }\end{array}$} & \multirow[t]{2}{*}{$\mathrm{n}$} & \multicolumn{4}{|c|}{ Survivin } & \multicolumn{4}{|c|}{ Bcl-2 } \\
\hline & & Positive & Negative & $\chi^{2}$ & P-value & Positive & Negative & $\chi^{2}$ & P-value \\
\hline Age (years) & & & & 0.534 & 0.465 & & & 0.061 & 0.806 \\
\hline$\geq 60$ & 30 & 16 & 14 & & & 15 & 15 & & \\
\hline$<60$ & 32 & 20 & 12 & & & 17 & 15 & & \\
\hline Gender & & & & 0.066 & 0.798 & & & 0.026 & 0.871 \\
\hline Male & 44 & 26 & 18 & & & 23 & 21 & & \\
\hline Female & 18 & 10 & 8 & & & 9 & 9 & & \\
\hline Histological type & & & & 2.268 & 0.322 & & & 0.753 & 0.252 \\
\hline Squamous cell carcinoma & 40 & 26 & 14 & & & 20 & 20 & & \\
\hline Adenocarcinoma & 18 & 8 & 10 & & & 12 & 6 & & \\
\hline Large-cell lung cancer & 4 & 2 & 2 & & & 1 & 3 & & \\
\hline Tumor diameter $(\mathrm{cm})$ & & & & 0.147 & 0.701 & & & 0.625 & 0.429 \\
\hline$\geq 5$ & 34 & 19 & 15 & & & 16 & 18 & & \\
\hline$<5$ & 28 & 17 & 11 & & & 16 & 12 & & \\
\hline Differentiation & & & & 5.534 & 0.019 & & & 0.003 & 0.960 \\
\hline High and moderate & 37 & 17 & 20 & & & 19 & 18 & & \\
\hline Poor & 25 & 19 & 6 & & & 13 & 12 & & \\
\hline Lymph node metastasis & & & & 4.324 & 0.038 & & & 0.041 & 0.840 \\
\hline Without & 24 & 10 & 14 & & & 12 & 12 & & \\
\hline With & 38 & 26 & 12 & & & 20 & 18 & & \\
\hline TNM stage & & & & 7.088 & 0.008 & & & 4.218 & 0.047 \\
\hline Stage I + II & 33 & 14 & 19 & & & 13 & 20 & & \\
\hline Stage III + IV & 29 & 22 & 7 & & & 19 & 10 & & \\
\hline
\end{tabular}

immuno-positive stained, brown-yellow vesicles varied in size. A relatively decreased expression of survivin and Bcl-2 was found in the tumor adjacent tissues in the lung. The percentage of survivin-positive cases in NSCLC tissues was significantly higher than that in tumor adjacent tissues (58.06 vs. 10\%; $\mathrm{P}<0.01$ ) (Table I). Likewise, the percentage of Bcl-2-positive cases in NSCLC tissues was significantly higher than that in tumor adjacent tissues (51.61 vs. 6.67\%; P<0.01) (Table I).

Relationship between survivin and Bcl-2 expression and clinicopathological features of NSCLC. Survivin expression in NSCLC tissues was found to be closely correlated with tumor differentiation, lymph node metastasis and TNM stage $(\mathrm{P}<0.05)$, whereas no significant differences were found between the survivin expression and patient age, gender, histological subtype or tumor size $(\mathrm{P}>0.05)$. Bcl-2 expression was associated with TNM stage $(\mathrm{P}<0.05)$, while no statistical differences were detected between the $\mathrm{Bcl}-2$ expression and patient age, gender, histological subtype, tumor size, differentiation or lymph node metastasis ( $\mathrm{P}>0.05)$ (Table II).
Association between survivin and Bcl-2 expression in NSCLC tissues. As shown in Table III, the percentage of Bcl-2-positive cells in NSCLC tissues with survivin-positive or survivin-negative samples was $63.88 \%(23 / 36)$ or $34.62 \%$ (9/26), respectively. Spearman analysis demonstrated that the expression of survivin and Bcl-2 in NSCLC tissues was closely correlated ( $\mathrm{rs}=0.289 ; \mathrm{P}<0.05)$.

Relationship between survivin and Bcl-2 expression in NSCLC tissues and prognosis. Kaplan-Meier survival curves showed that the expression of survivin and $\mathrm{Bcl}-2$ was negatively correlated with survival rate. The 5-year survival rate for NSCLC patients with survivin-positive cells was significantly reduced, as compared to that in patients with survivin-negative cells (16.7\%, 6/36 vs. 53.8\%, 14/26; P<0.05) (Fig. 3). Similar results were obtained for Bcl-2 expression, as the 5-year survival rate for NSCLC patients with Bcl-2-positive cells was significantly reduced compared to that of patients with Bcl-2-negative cells (18.8\%, 6/32 vs. 46.7\%, 14/30; P<0.05) (Fig. 4). Moreover, the 5-year survival rate for NSCLC patients with positivity 
Table III. Association between survivin and Bcl-2 expression in NSCLC tissues.

\begin{tabular}{lccc}
\hline Survivin & \multicolumn{2}{c}{ Bcl-2 } & Total \\
\cline { 2 - 3 } & Positive & Negative & \\
\hline Positive & 23 & 13 & 36 \\
Negative & 9 & 17 & 26 \\
Total & 32 & 30 & 62 \\
\hline
\end{tabular}

for both survivin and Bcl-2 was statistically lower than that in patients with negativity for both survivin and Bcl-2 (8.7\%, $2 / 23$ vs. 58.8\%, 7/17; P<0.05) (Fig. 5).

\section{Discussion}

The tumorigenesis and development of lung cancer is a complex process characterized by an imbalance in cell proliferation and apoptosis that is regulated by various genes. Impairment of apoptosis, caused by inactivation or disequilibrium of various apoptosis mediators, is recognized as an essential mechanism involved in this process $(9,10)$. Apoptosis, also known as programmed cell death, is a critical process of normal biological function, and has been highly conserved throughout evolution. Apoptosis mediates the clearance of damaged, aging or excessive cells, and is crucial for the maintenance of normal tissue structure. Abnormal proliferation of tumor cells may occur when apoptotic cell death is inhibited, which ultimately supports tumorigenesis, development, invasion and metastasis (11).

Survivin is the smallest inhibitor of apoptosis protein (IAP) family member (by molecular weight), but is the most efficient in inhibitory capacity. First identified in 1997 by Ambrosini et al (12), up-regulated survivin expression has since been detected in a large proportion of transformed cell lines and human tumor tissues. Notably, relatively low or no survivin expression has been found in tumor adjacent tissues and most normal adult tissues. Survivin expression levels have been determined to be closely correlated with tumorigenesis, development and prognosis $(13,14)$.

Monzó et al were the first to examine survivin expression in NSCLC cases using RT-PCR. Increased survivin expression was observed in 71 of the 83 NSCLC cases examined (85.5\%), while only $12 \%$ of the cases also showed survivinpositive expression in normal lung tissues (15). Subsequently, Hofmann et al evaluated survivin gene and protein expression in specimens obtained from NSCLC patients (16 cases of adenocarcinoma and 18 cases of squamous cell carcinoma) using RT-PCR and western blotting, respectively. As compared to those in non-tumor tissues, the mRNA levels of survivin were found to be enhanced in all the tumor specimens, demonstrating that survivin not only contributes to tumor escape from the inherent surveillance system, but also plays a critical role in the development and progression of lung cancer through suppressing apoptosis of lung cancer cells (16). Ikehara $e t$ al performed immunohistochemistry on tumor specimens $(<2 \mathrm{~cm})$ obtained from patients with small adenocarcinoma of

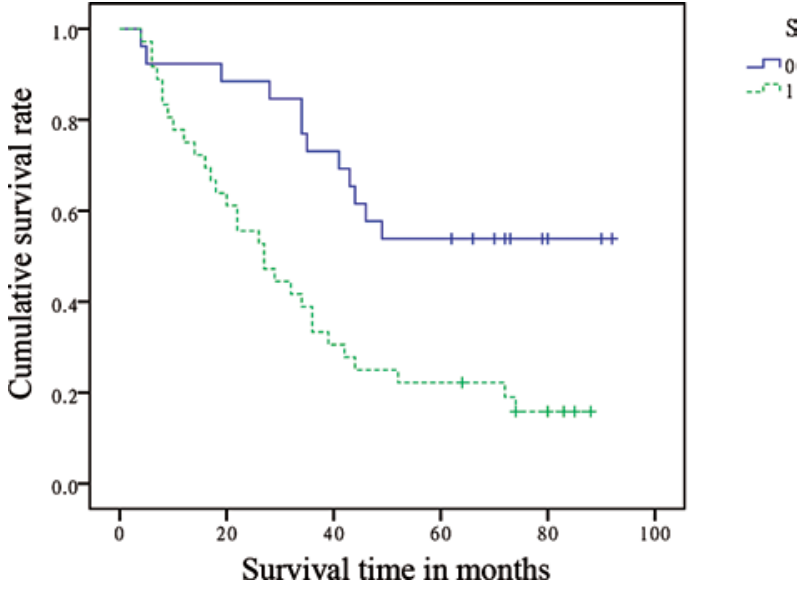

Figure 3. Kaplan-Meier survival curve for survivin. Group 0, negative for expression of survivin (solid line). Group 1, positive for expression of survivin (dotted line).

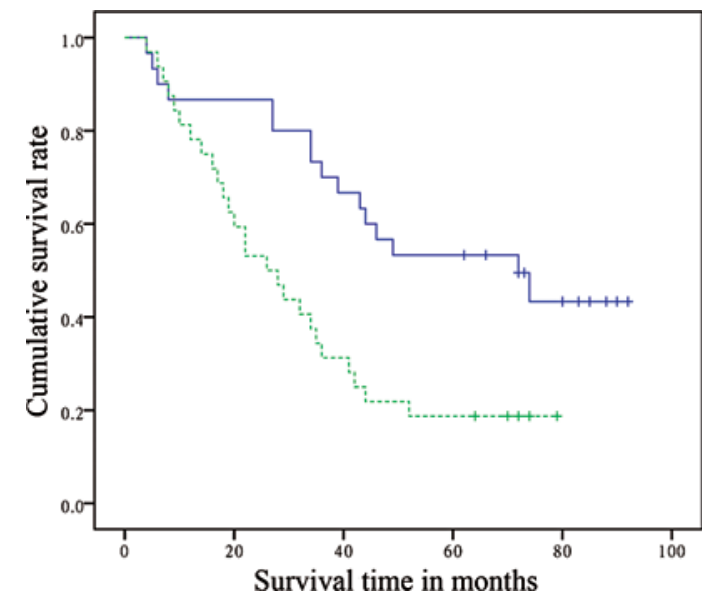

Figure 4. Kaplan-Meier survival curve for Bcl-2. Group 0, negative for expression of Bcl-2 (solid line). Group 1, positive for expression of Bcl-2 (dotted line).

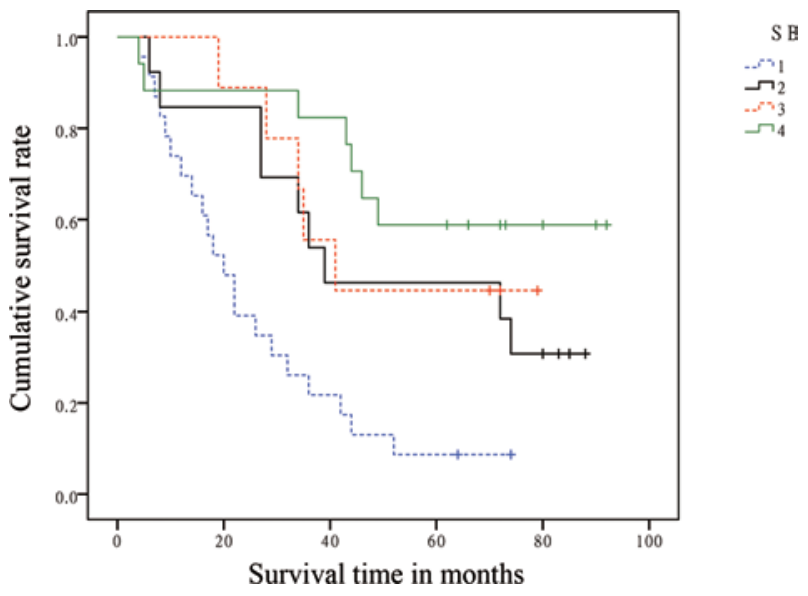

Figure 5. Combined Kaplan-Meier survival curve. Group 1, positive for expression of both survivin and Bcl-2 (dotted line). Group 2, positive for expression of survivin and negative for expression of $\mathrm{Bcl}-2$ (solid line). Group 3 , positive for expression of Bcl-2 and negative for expression of survivin (dotted line). Group 4, negative for expression of both survivin and Bcl-2 (solid line) 
the lung, and found that 38 patients had tumors consisting of $\leq 10 \%$ survivin-positive cells (negative group) and 41 patients had tumors consisting of $>10 \%$ survivin-positive cells (positive group). The incidence of venous invasion was significantly higher in the survivin-positive group, while the overall survival of survivin-positive patients was significantly worse than that of survivin-negative patients, suggesting that expression of survivin in tumor cells was a poor prognostic factor in patients with small adenocarcinoma of the lung (17). Our results revealed that the percentage of samples of survivin-positive expression in NSCLC tissues was significantly higher than the percentage in tumor adjacent tissues $(\mathrm{P}<0.05)$. In addition, survivin expression was closely correlated with tumor differentiation, lymph node metastasis and TNM stages $(\mathrm{P}<0.05)$, whereas no significant differences were found between survivin expression and patient age, gender, histological subtype or tumor size $(\mathrm{P}>0.05)$. In addition, the 5-year survival rate for NSCLC patients with survivin-positive expression was significantly reduced, as compared to that in patients with survivin-negative expression $(16.7 \%, 6 / 36$ vs. $53.8 \%, 14 / 26 ; \mathrm{P}<0.05)$. These findings suggest that tumor tissues with survivin-positive expression may be quite aggressive and lead to poor prognosis.

The Bcl-2 gene was identified by Tsujimoto in 1984 from a study of chromosomal breakpoints of follicular B cell lymphomas (18). Bcl-2 has since become widely accepted as one of the most important apoptosis-suppressing genes. Overexpression of Bcl-2 has no influence on cell proliferation and division, but prolongs tumor cell survival duration and suppresses tumor cell apoptosis. Furthermore, Bcl-2 has been shown to contribute to tumor escape from the inherent surveillance system by promoting the accumulation of mutant genes, and its involvement in tumorigenesis and development has been demonstrated $(19,20)$. Consistent with previous reports $(21,22)$, our results showed that the Bcl-2-positive rate was $51.6 \%$ in NSCLC tissues, which was significantly higher than that detected in tumor adjacent tissues $(\mathrm{P}<0.05)$. Bcl-2 expression was associated with TNM stage $(\mathrm{P}<0.05)$, while no statistical differences were detected between Bcl-2 expression and patient age, gender, histological subtype, tumor size, differentiation or lymph node metastasis ( $\mathrm{P}>0.05)$. Five-year follow-up revealed that Bcl-2 expression was negatively correlated with postoperative survival duration, as patients with Bcl-2-negative expression showed longer post-operative survival time than those with Bcl-2-positive expression $(\mathrm{P}<0.01)$. Based on these observations, we conclude that $\mathrm{Bcl}-2$ plays an essential role in the development, invasion and metastasis of NSCLC, and may be used as a biomarker for evaluating the biological behavior and prognosis of NSCLC.

Survivin and Bcl-2 occupy a central place in the IAP family and apoptosis-suppressing Bcl-2 family, respectively. Their correlations with tumorigenesis and development of malignant tumors have garnered significant research attention. Al-Joudi et al analyzed the survivin and Bcl-2 expression in 382 cases of invasive ductal carcinoma of the breast and found that the $\mathrm{Bcl}-2$-positive rate in patients with survivin-positive or -negative expression was $54.62 \%$ (142/260) and $20.49 \%$ (25/122), respectively, demonstrating the positive correlation between survivin and Bcl-2 expression $(\mathrm{P}<0.05)(23)$. Han et al analyzed the survivin and $\mathrm{Bcl}-2$ expression in 87 cases of NSCLC and found a close correlation between them. Moreover, dual survivin and Bcl-2 expression has been associated with lymph node metastasis (24). In this study, we found that the percentage of Bcl-2-positive cases in NSCLC tissues with survivin-positive or -negative was $63.88 \%$ (23/36) or $34.62 \%$ (9/26), respectively. Spearman analysis demonstrated that the expression of survivin and Bcl-2 in NSCLC tissues is closely correlated $(\mathrm{P}<0.05)$. Furthermore, the 5-year survival rate for NSCLC patients with dual survivin- and Bcl-2-positivity was statistically lower than that in patients with dual survivin- and Bcl-2-negativity $(8.7 \%, 2 / 23$ vs. 46.7\%, 14/30; $\mathrm{P}<0.05)$. These results indicated that survivin and $\mathrm{Bcl}-2$ may act synergistically to promote occurrence, development, invasion and metastasis of NSCLC.

Survivin gene is located at $17 \mathrm{q} 25$, while the Bcl-2 gene is located at $18 \mathrm{q} 21$. Although the chromosome locations of the genes are different, it is possible that certain transcription regulatory elements may functionally collude to mediate the co-expression of survivin and Bcl-2 in tumor tissues. On the other hand, both survivin and $\mathrm{Bcl}-2$ genes are regulated by GC-rich promoters that lack a TATA box. Transcription of survivin and Bcl-2 occurs in cells with high proliferation activity, implying these genes may share a common transcriptional pathway. Survivin produces its anti-apoptosis function by inhibiting caspase- 3 and caspase- 7 activities. Bcl-2, which serves as the upstream factor of the caspase cascade reaction, suppresses cell apoptosis through blocking the release of cytochrome $\mathrm{C}$ from mitochondria. Survivin and Bcl-2 regulate different anti-apoptotic pathways, but it is possible that the two mechanisms are convergent and together contribute to the development and progression of NSCLC. Nevertheless, the detailed mechanism requires further clarification $(25,26)$.

Collectively, lines of evidence have indicated that survivin and Bcl-2 play essential roles in tumorigenesis, development, invasion and metastasis of malignant tumor; however, a significant amount of studies have yielded discrepant findings. For example, Kaur et al analyzed the survivin and Bcl-2 expression by immunohistochemistry in 138 tumor tissues of patients with prostatic adenocarcinomas. Spearman analysis revealed that no correlation existed between survivin and Bcl-2 expression (27). Jeong et al also showed that survivin expression was not related to Bcl-2 expression in urothelial cancer of the upper urinary tract. In particular, survivin-positive expression was found to be associated with poor prognosis, shorter overall survival time and high frequency of tumor recurrence, whereas Bcl-2 expression was not associated with the clinical prognosis (28). Zhu et al reported that survivin expression was not related to $\mathrm{Bcl}-2$ expression in cervical carcinoma tissues. Additionally, the levels of survivin and Bcl-2 were negatively correlated with prognosis. In that study, patients with survivinand Bcl-2-positive expression showed a shorter overall survival period than those with survivin- and Bcl-2-negative expression $(\mathrm{P}<0.01)(29)$. Kren et al further demonstrated that survivin was negatively associated with Bcl-2 expression in NSCLC tissues. Patients with survivin-positive expression were shown to have poor prognosis and a shorter overall survival period, as compared to patients with survivin-negative expression $(\mathrm{P}<0.01)$. Moreover, patients with $\mathrm{Bcl}-2$-positive expression showed better prognosis and overall survival than those with Bcl-2-negative expression $(\mathrm{P}<0.01)$ (30). Therefore, the study of survivin and $\mathrm{Bcl}-2$ promotes the further understanding of 
the tumorigenesis, development, invasion and metastasis of NSCLC, and may provide a novel approach for the prevention and control of lung cancer.

\section{Acknowledgements}

This study was supported by grants from the Natural Science Foundation of Fujian Province (no. 2008J0284), and the Science and Technology Project of Fujian Provincial Department of Education (no. JA09121).

\section{References}

1. Ferlay J, Shin HR, Bray F, Forman D, Mathers C and Parkin DM: Estimates of worldwide burden of cancer in 2008. GLOBOCAN 2008. Int J Cancer 127: 2893-2917, 2010.

2. Sato M, Shames DS, Gazdar AF and Minna JD: A translational view of the molecular pathogenesis of lung cancer. J Thorac Oncol 2: 327-343, 2007.

3. Yang Y, Zhu J, Gou H, Cao D, Jiang M and Hou M: Clinical significance of Cox-2, survivin and Bcl-2 expression in hepatocellular carcinoma (HCC). Med Oncol 28: 796-803, 2011.

4. Athanassiadou AM, Patsouris E, Tsipis A, Gonidi M and Athanassiadou P: The significance of survivin and Nectin-4 expression in the prognosis of breast carcinoma. Folia Histochem Cytobiol 49: 26-33, 2011.

5. Gan XL, Li JK, Yu TH, Zhang Y and Hu LN: High expressions of bcl-2 and survivin, and decreased apoptosis in uterine cervical carcinosarcoma compared to cervical squamous cell carcinoma. Arch Gynecol Obstet 284: 175-181, 2011.

6. Naruke T: Results of resected lung cancer based on the new UICC TNM classification, 5th edition. Gan To Kagaku Ryoho 24: 2289-2295, 1997.

7. Travis WD, Brambilla E, Muller-Hermelink HK and Harris CC (eds.): World Health Organization Classification of Tumours Pathology and Genetics of Tumours of the Lung, Pleura and Heart. IARC Press, Lyon, 2004.

8. Sinicrope FA, Ruan SB, Cleary KR, Stephens LC, Lee JJ and Levin B: bcl-2 and p53 oncoprotein expression during colorectal tumorigenesis. Cancer Res 55: 237-241, 1995.

9. Shivapurkar N, Reddy J, Chaudhary PM and Gazdar AF: Apoptosis and lung cancer: a review. J Cell Biochem 88: 885-898, 2003.

10. Xu CX, Jin H and Cho MH: Apoptosis and apoptosis-based therapy in lung cancer. Anticancer Agents Med Chem 9: 952-957, 2009.

11. Hengartner MO: The biochemistry of apoptosis. Nature 407: 770-776, 2000

12. Ambrosini G, Adida C and Altieri DC: A novel anti-apoptosis gene, survivin, expressed in cancer and lymphoma. Nat Med 3: 917-921, 1997.

13. Youssef NS, Hewedi IH and Abd Raboh NM: Immunohistochemical expression of survivin in breast carcinoma relationship with clinicopathological parameters, proliferation and molecular classification. J Egypt Natl Canc Inst 20: 348-357, 2008 .
14. Kalliakmanis JG, Kouvidou Ch, Latoufis C, et al: survivin expression in colorectal carcinomas: correlations with clinicopathological parameters and survival. Dig Dis Sci 55: 2958-2964, 2010.

15. Monzó M, Rosell R, Felip E, et al: A novel anti-apoptosis gene: Re-expression of survivin messenger RNA as a prognosis marker in non-small-cell lung cancers. J Clin Oncol 17: 2100-2104, 1999.

16. Hofmann HS, Simm A, Hammer A, Silber RE and Bartling B: Expression of inhibitors of apoptosis (IAP) proteins in non-small cell human lung cancer. J Cancer Res Clin Oncol 128: 554-560, 2002.

17. Ikehara M, Oshita F, Kameda Y, et al: Expression of survivin correlated with vessel invasion is a marker of poor prognosis in small adenocarcinoma of the lung. Oncol Rep 9: 835-838, 2002.

18. Tsujimoto Y, Gorham J, Cossman J, Jaffe E and Croce CM: The $\mathrm{t}(14 ; 18)$ chromosome translocations involved in B-cell neoplasms result from mistakes in VDJ joining. Science 229: 1390-1393, 1985.

19. Llambi F and Green DR: Apoptosis and oncogenesis: give and take in the BCL-2 family. Curr Opin Genet Dev 21: 12-20, 2011

20. Brambilla E and Gazdar A: Pathogenesis of lung cancer signalling pathways: roadmap for therapies. Eur Respir J 33: 1485-1497, 2009.

21. Wang DF, Zeng CG, Lin YB, Hou JH and Zhu ZH: Expression and clinical significance of apoptosis-related oncogenes in stage I-II non-small cell lung cancer. Ai Zheng 25: 359-362, 2006.

22. Shibata Y, Hidaka S, Tagawa Y and Nagayasu T: Bcl-2 protein expression correlates with better prognosis in patients with advanced non-small cell lung cancer. Anticancer Res 24: 1925-1928, 2004

23. Al-Joudi FS, Iskandar ZA and Imran AK: Correlations in survivin expression with the expression of p53 and bcl-2 in invasive ductal carcinoma of the breast. Southeast Asian J Trop Med Public Health 38: 904-910, 2007.

24. Han PH, Li XJ, Qin H, Yao J, DU N and Ren H: Upregulation of survivin in non-small cell lung cancer and its clinicopathological correlation with 553 and Bcl-2. Xi Bao Yu Fen Zi Mian Yi Xue Za Zhi 25: 710-713, 2009

25. Chen ZX and Pervaiz S: Involvement of cytochrome c oxidase subunits $\mathrm{Va}$ and $\mathrm{Vb}$ in the regulation of cancer cell metabolism by Bcl-2. Cell Death Differ 17: 408-420, 2010.

26. Altieri DC: New wirings in the survivin networks. Oncogene 27: 6276-6284, 2008.

27. Kaur P, Kallakury BS, Sheehan CE, Fisher HA, Kaufman RP Jr and Ross JS: survivin and Bcl-2 expression in prostatic adenocarcinomas. Arch Pathol Lab Med 128: 39-43, 2004.

28. Jeong IG, Kim SH, Jeon HG, Kim BH, Moon KC, Lee SE and Lee E: Prognostic value of apoptosis-related markers in urothelial cancer of the upper urinary tract. Hum Pathol 40: 666-677, 2009.

29. Zhu H, Hu Y, Shuai CX, Shi ZZ and Zhang HX: Expression of survivin, bcl-2 in cervical carcinoma and its association with HPV16/18 infection. Zhonghua Yi Xue Za Zhi 90: 469-473, 2010.

30. Kren L, Brazdil J, Hermanova M, Goncharuk VN, Kallakury BV Kaur P and Ross JS: Prognostic significance of anti-apoptosis proteins survivin and bcl-2 in non-small cell lung carcinomas: a clinicopathologic study of 102 cases. Appl Immunohistochem Mol Morphol 12: 44-49, 2004. 